ORIGINAL ARTICLE

\title{
High-level resistance to gentamicin: genetic transfer between Enterococcus faecalis isolated from food of animal origin and human microbiota
}

\author{
M. Sparo ${ }^{1,2 *}$, L. Urbizu ${ }^{2,3 *}$, M.V. Solana ${ }^{2}$, G. Pourcel ${ }^{1}$, G. Delpech ${ }^{1}$, A. Confalonieri ${ }^{2,3}$, M. Ceci ${ }^{2}$ and \\ S.F. Sánchez Bruni ${ }^{2,3}$ \\ 1 Clinical Microbiology Division, School of Medicine, Universidad Nacional del Centro de la Provincia de Buenos Aires (UNCPBA), Olavarría, \\ Argentina \\ 2 Laboratory of Pharmacology, Faculty of Veterinary Medicine, UNCPBA, Tandil, Argentina \\ 3 Argentinian Council of Science and Technology (CONICET), Buenos Aires, Argentina
}

\section{Keywords}

conjugative plasmid, Enterococcus faecalis, human microbiota, mice, resistance.

\section{Correspondence}

Monica Sparo, Clinical Microbiology Division, School of Medicine, UNCPBA, Buenos Aires, Argentina.

E-mail: msparo@vet.unicen.edu.ar

*Both authors contributed equally to this work

2011/1076: received 29 June 2011, revised 28 September 2011 and accepted 10 November 2011

doi:10.1111/j.1472-765X.2011.03182.x

\begin{abstract}
Aims: To investigate the in vivo gene transfer of high-level gentamicin resistance (HLRG) from Enterococcus faecalis isolated from the food of animal origin to a human isolate, using a mouse model of intestinally colonized human microbiota.

Methods and Results: In vitro study: The presence of plasmids involved in HLRG coding was investigated. After the conjugation experiment, the recipient strain, Ent. faecalis JH2-SS, acquired a plasmid responsible for HLRG [minimal inhibitory concentration (MIC) $>800 \mu \mathrm{g} \mathrm{ml}^{-1}$ ], in a similar position to the donor cells. In vivo study: Seven BALB/c mice were dosed with ceftriaxone $\left(400 \mathrm{mg} \mathrm{kg}^{-1}\right)$ and then inoculated with a dilution of 1/100 of human faeces (HFc). After $72 \mathrm{~h}$, Ent. faecalis JH2-SS (recipient) was inoculated and then, after a further $72 \mathrm{~h}$, the animals were given Ent. faecalis CS19, isolated from the food of animal origin, involved in HLRG (donor). The presence of transconjugant strains in HFc was subsequently recorded on a daily basis until the end of the experiment. The clonal relationship between Ent. faecalis and Escherichia coli in faeces was assessed by RAPD-PCR. Both the in vitro and in vivo studies showed that the receptor strain acquired a plasmid responsible for HLRG (MICs $>800 \mu \mathrm{g} \mathrm{ml}^{-1}$ ), which migrated with a similar relative mobility value. Transconjugant strains were detected from $24 \mathrm{~h}$ after the donor strain inoculation and persisted until the end of the experiment.

Conclusions: The in vivo gene transfer of HLRG from Ent. faecalis strains, isolated from the food of animal origin, to human microbiota has been demonstrated in a mouse model.

Significance and Impact of the Study: The complexity found on the therapeutic responses of invasive infectious diseases caused by Ent. faecalis facilitates the assessment of food of animal origin as a resistant pathogen reservoir. In addition, this study may contribute to the understanding of antimicrobials' resistance gene transfer between Ent. faecalis strains from food and human GI tract.
\end{abstract}

\section{Introduction}

The relevance of the genus Enterococcus as an emerging pathogen associated with health care is based on (i) its natural resistance to conventional antimicrobial agents
(ATM) and (ii) its ability to acquire resistance from other bacteria, resulting in serious problems in terms of ATM therapy. Enterococci are intrinsically resistant to many ATM based in chromosomal genes, which typically are nontransferrable. Specifically, for aminoglycosides, the 
low-level resistance reported as minimal inhibitory concentration (MIC) for gentamicin was $4-128 \mu \mathrm{g} \mathrm{ml}^{-1}$ and for streptomycin, 64-512 $\mu \mathrm{g} \mathrm{ml}^{-1}$ (Leclercq 1994). Early in 1970, it was reported that the highest levels of resistance to both streptomycin (MIC $>2000 \mu \mathrm{g} \mathrm{ml}^{-1}$ ) and gentamicin (HLRG) (MIC $>500 \mu \mathrm{g} \mathrm{m}^{-1}$ ) were found in Enterococcus faecalis strains. HLRG was mainly because of the presence of the bifunctional aminoglycoside-modifying enzyme, Aac6'-Aph2". Other research confirmed that HLRG is prevalent in the strains of both Ent. faecalis and Enterococcus faecium (Moellering et al. 1970; Ferretti et al. 1986). More recently, it has been reported that HLRG in some strains of enterococci is linked to transposable elements with plasmidic localization or chromosomal integration (Predari et al. 1999; Ronconi and Merino 2000).

Enterococci are predominantly associated with mammals, although they have also been found in water and vegetables (Sparo and Mallo 2001; Foulquié et al. 2006). Moreover, enterococci may colonize raw food (milk and meat) through intestinal residues or environmental contamination.

Some strains of Ent. faecalis may be considered probiotics with beneficial effects to the host (Castro et al. 2007). In contrast, other Ent. faecalis strains may have some pathogenicity determinants and also the capability of acquiring and transferring ATM resistance genes. The latter play a pivotal role in several invasive diseases including intra-abdominal and pelvic abscesses, endocarditis and occasionally meningitis or pneumonia (Gentile et al. 1995; Maschieto et al. 2004). Interestingly, ATM multiresistant strains of enterococci used in human medicine have been isolated from nonhospital environments such as poultry faeces (Hayes et al. 2004). In this context, controlled in vitro studies have been developed to evaluate the gene transfer to intestinal microbiota (Clewell 1990; Donabedian et al. 2003). However, in vivo studies to confirm the in vitro evidence for resistance gene transfer are required. In this context, the main goal of this research was to demonstrate the transfer of HLRG genes from Ent. faecalis isolated from the food of animal origin to mice colonized with human microbiota.

\section{Materials and methods}

\section{Bacterial strains}

Donor strains: Nine strains of Ent. faecalis (CS17-CS26) were isolated from the food of animal origin (CS17-CS21 - ground meat beef, CS22-CS25 - artisanal crafted sausages and CS26 - cattle cheese), containing HLRG gene (MIC gentamicin $>800 \mu \mathrm{g} \mathrm{ml}^{-1}$ ). These strains were provided by the Laboratory of Pharmacology, Faculty of Veterinary Medicine, UNCPBA, Argentina. Recipient strain: Human Ent. faecalis JH2-SS strain (obtained from the Michael S. Gilmore collection, Oklahoma University, USA) without plasmids, but resistant to streptomycin (MIC: $\quad 500 \mu \mathrm{g} \mathrm{ml}^{-1}$ ) and spectinomycin (MIC: $500 \mu \mathrm{g} \mathrm{ml}^{-1}$ ) by spontaneous mutation, was used in the in vitro assay (Yagi and Clewell 1980).

\section{Gene transfer experiments}

In vitro assays. The presence of plasmids involved in HLRG coding and plasmid curing was assessed using an assay based on the protocol previously described by RuizBarba et al. (1991) and Sparo et al. (2006).

Plasmid curing. Briefly, after culture in brain heart infusion (BHI) broth, aliquots of $10 \mathrm{ml}$ Ent. faecalis from the food of animal origin (MIC gentamicin $>800 \mu \mathrm{g} \mathrm{ml}^{-1}$ ) were adjusted to a viable count of $10^{5} \mathrm{CFU} \mathrm{ml}{ }^{-1}$. Then, after the addition of novobiocin (Sigma-Aldrich, USA) at several concentrations between $0 \cdot 125$ to $16 \mu \mathrm{g} \mathrm{ml}^{-1}$ and pronase (Sigma-Aldrich) at a final concentration of $0.5 \mathrm{~g} \mathrm{l}^{-1}$, cultures were incubated for $72 \mathrm{~h}$ at $35^{\circ} \mathrm{C}$. Cells incubated without added novobiocin were used as a control. After incubation, cells treated with the highest concentration of novobiocin displaying visible growth were diluted in sterile saline and plated onto BHI agar. After overnight incubation at $35^{\circ} \mathrm{C}$, replicate plating was carried out to BHI agar and BHI agar containing gentamicin $\left(500 \mu \mathrm{g} \mathrm{ml}^{-1}\right)$. Colonies that grew only in BHI agar were picked and transferred to BHI broth. These bacteria were stored at $-20^{\circ} \mathrm{C}$ in $\mathrm{BHI}$ infusion containing $20 \% \mathrm{v} / \mathrm{v}$ glycerol until used in the conjugation experiments. Plasmid profiles of Ent. faecalis strains from the food of animal origin were analysed before and after the curing experiments as described below.

Conjugation. For conjugation assessment, a 1:10 ratio of donor/recipient cells was used. Strains from the food (donors) and human Ent. faecalis JH2-SS (recipient) were mixed in BHI broth. After incubation for $5 \mathrm{~h}$ at $35^{\circ} \mathrm{C}$, streptomycin $\left(300 \mu \mathrm{g} \mathrm{ml}^{-1}\right.$; Sigma-Aldrich) was added and the incubation continued for a further $9 \mathrm{~h}$ at $35^{\circ} \mathrm{C}$. Samples were then centrifuged at $2600 \mathrm{~g}\left(4^{\circ} \mathrm{C}\right)$ by $10 \mathrm{~min}$, and the resultant sediments were suspended in $0.5 \mathrm{ml}$ of BHI broth. Dilutions of this suspension were made in BHI agar supplemented with streptomycin $\left(300 \mu \mathrm{g} \mathrm{ml}^{-1}\right)$ and gentamicin $\left(500 \mu \mathrm{g} \mathrm{ml}^{-1}\right)$. All samples were incubated for $48 \mathrm{~h}$ at $35^{\circ} \mathrm{C}$ in order to determine the viable counts $\left(\mathrm{CFU} \mathrm{m}{ }^{-1}\right)$. Plasmid content and genetic identity were determined as described in the following sections. Three control groups were set up to eliminate possible alternative gene-transfer mechanisms as follows: (i) Ent. faecalis isolates from the animal food (donor) were treated with chloroform and DNase (Sigma-Aldrich) before being mixed with recipient cells, 
(ii) recipient bacteria were incubated with supernatants from the donor strain cultures and (iii) donor and recipient bacteria were co-cultured in BHI broth containing gentamicin $\quad\left(500 \mu \mathrm{g} \mathrm{ml}^{-1}\right) \quad$ and streptomycin $\left(300 \mu \mathrm{g} \mathrm{ml}^{-1}\right)$. Conjugation frequency was calculated as the number of transconjugant cells (expressed as $\mathrm{CFU} \mathrm{ml} \mathrm{m}^{-1}$ ) divided by the number of donor cells, where transconjugant cells were considered as those which exhibited growth in BHI agar supplemented with gentamicin and streptomycin.

Plasmid content. The Qiagen ${ }^{\circledR}$ (Plasmid Midi kit; Qiagen, CA, USA) method modified as previously described (Sparo et al. 2006) was utilized to analyse the plasmid content. The same procedure was also used with Escherichia coli strain V517 to provide a plasmid content reference range with the following values: $56 \cdot 4,7 \cdot 5,5 \cdot 3,4 \cdot 0$, $3 \cdot 1,2 \cdot 8$ and $2 \cdot 2 \mathrm{kbp}$.

RAPD-PCR of enterococci strains. The RAPD-PCR of Ent. faecalis CS17-CS26 and Ent. faecalis JH2-SS strains was performed according to Sparo et al. (2008). The primers used were D8635 (5' GAG CGG CCA AAG GGA GCA GAC 3') and M13 (5' GAG GGT GGC GGT TCT $\left.3^{\prime}\right)$. The clonal relationship was set up by the software RAPDistance 1.04 Software Package (Australian National University). The strain isolated from the food of animal origin with lowest conjugation frequency per donor cell was used for the HLRG in vivo transfer study.

In vivo assay. Replacement of the intestinal microbiota. Seven 8-week-old BALB/c mice (approximately $25 \mathrm{~g}$ live body weight) were dosed intraperitoneally twice, $24 \mathrm{~h}$ apart, with $400 \mathrm{mg} \mathrm{kg}^{-1}$ ceftriaxone (Roche, Vaud, Switzerland), in order to decrease the coliform population in the intestine. Sixty hours after this ATM treatment, mice were inoculated by the intragastric (IG) route with human faeces $(\mathrm{HFc}$ ) diluted 1/100 in sterile deionized water. HFc was obtained from a 6-month-old child, still on milk, who had not received any previous ATM treatment and had no detectable enterococci population, on the evidence of screening in selective and nonselective cultures (BHI agar and bile-esculin-azide agar, respectively). For the analysis of coliform populations, mice were sampled at 24 and $0 \mathrm{~h}$ before treatment, then again 24 and $48 \mathrm{~h}$ after treatment with ceftriaxone. Further samples were taken after HFc inoculation at the end of the experiment (15 days). Coliform and enterococcal viable counts (CFU g faeces) were determined in EMB-Levine agar and bile-esculinazide agar, respectively, by the plate count method.

Intestinal tract implantation of recipient and donor strains. Seventy-two hours after inoculation of $\mathrm{HFc}$, mice were dosed orally with the recipient strain, Ent. faecalis JH2-SS $\left(1.5 \times 10^{8} \mathrm{CFU}\right)$, and the subsequent presence of this strain in faeces was investigated at 24 and $48 \mathrm{~h}$ after treatment. At $72 \mathrm{~h}$, the donor isolate that demonstrated the lowest conjugation frequency in vitro was inoculated IG $\left(1.5 \times 10^{8} \mathrm{CFU}\right)$. Then, for the following 8 days, faeces were collected to investigate the presence of donor, recipient and transconjugant Ent. faecalis strains. Finally, the distribution of these strains was determined in intestinal tissue removed from the mice at postmortem following humane sacrifice.

Enterobacteria and enterococci strain characterization. Enterobacterial phenotyping was performed using the API-20E (bioMérieux, France) system, whilst enterococci were identified according to the method previously described by Sparo et al. (2006, 2008).

RAPD-PCR studies. The clonal relationship of E. coli was determined by RAPD-PCR following the protocol reported by Cave et al. (1994). The primer used was the oligonucleotide 1274, 5'-AAG AGC CCG-3'. The RAPD$\mathrm{PCR}$, technique described on the in vitro assays, was used for the genetic characterization of Ent. faecalis transconjugants.

\section{Results}

\section{In vitro assay}

In Ent. faecalis strains with HLRG recovered from the food of animal origin, bacterial development was observed in cultures containing novobiocin concentrations of up to $4 \mu \mathrm{g} \mathrm{ml}^{-1}$. Plasmid curing was observed in all strains assayed which grew in BHI agar medium without gentamicin but did not develop in gentamicin-supplemented medium (Fig. 1, lanes 2 and 3 - Ent. faecalis

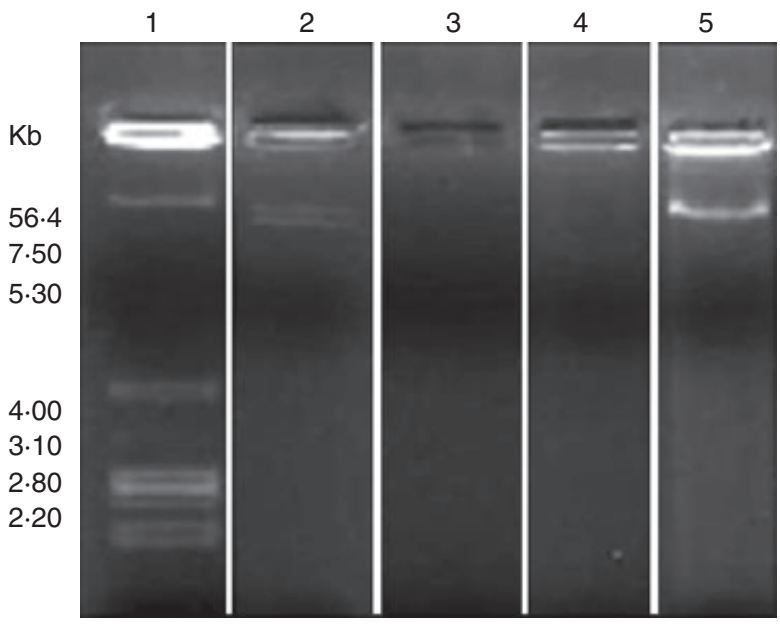

Figure 1 In vitro conjugation plasmid transference. Lane 1: plasmids of Escherichia coli V517, lane 2: Enterococcus faecalis CS19, lane 3: Ent. faecalis CS19 cured, lane 4: Ent. faecalis JH2-SS, lane 5: Ent. faecalis JH2-SS transconjugated. 
CS19 strain). However, Ent. faecalis strains that were not exposed to novobiocin retained the plasmid and successfully grew in gentamicin-supplemented agar. After the conjugation experiment, the recipient strain Ent. faecalis $\mathrm{JH} 2$-SS migrated with a relative mobility value (Rf) similar to that of the donor cells, indicating that plasmid incorporation was at a similar position (Fig. 1, lane 5 Ent. faecalis CS19), and the plasmid acquired by this strain was responsible for HLRG resistance (MIC $>800 \mu \mathrm{g} \mathrm{ml}^{-1}$ ). The frequency of transference of the plasmid by conjugation ranged from $2.9 \times 10^{-5}$ to $2.6 \times 10^{-3}$ per donor cell. Of the strains isolated from the food of animal origin, Ent. faecalis CS19 showed the lowest frequency of plasmid transfer.

\section{In vivo assay}

The enterobacterial population of the inoculated HFc contained E. coli $10^{8} \mathrm{CFU} \mathrm{g}^{-1}$ faeces. Before ceftriaxone administration, the enterobacterial population in mouse faeces was as follows: E. coli $10^{4}-10^{5} \mathrm{CFU} \mathrm{g}{ }^{-1}$ faeces, Enterobacter spp. $10^{3}-10^{4} \mathrm{CFU} \mathrm{g}{ }^{-1}$ faeces and Proteus spp. $10^{2}-10^{3} \mathrm{CFU} \mathrm{g}^{-1}$ faeces. After ceftriaxone administration, the faecal enterobacterial population changed as follows: E. coli was not detected, Enterobacter spp. $10^{2}-$ $10^{3} \mathrm{CFU} \mathrm{g}^{-1}$ faeces and Proteus spp. $10^{3}-10^{4} \mathrm{CFU} \mathrm{g}^{-1}$ faeces. Interestingly, $24 \mathrm{~h}$ after the administration of $\mathrm{HFc}$, mice were colonized with E. coli $\left(10^{5}-10^{6} \mathrm{CFU} \mathrm{g^{-1 }}\right.$ faeces). These changes on mice microbiota are illustrated in Fig. 2. Clonal heterogenicity of E. coli in mice faeces before inoculation of HMc was evidenced by the different electrophoretic profiles obtained by RAPD-PCR. However, a random selection of 8-10 colonies from EMBLevine agar cultures of mouse faeces sampled 9 days after HFc inoculation indicated that the E. coli population had a genetic profile identical to that of the implanted human E. coli. Colonization by the latter persisted in all the HFctreated mice until day $10( \pm 1)$ postinoculation. The recipient (JH2-SS) and the donor (CS19) Ent. faecalis strains were detected in mouse faeces from $24 \mathrm{~h}$ postinoculation and both persisted until the end of the experiment (Day 10; see Fig. 3). Transconjugant strains were detected at $24 \mathrm{~h}$ postdonor strain inoculation and, again, these persisted for the duration of the experiment, with numbers

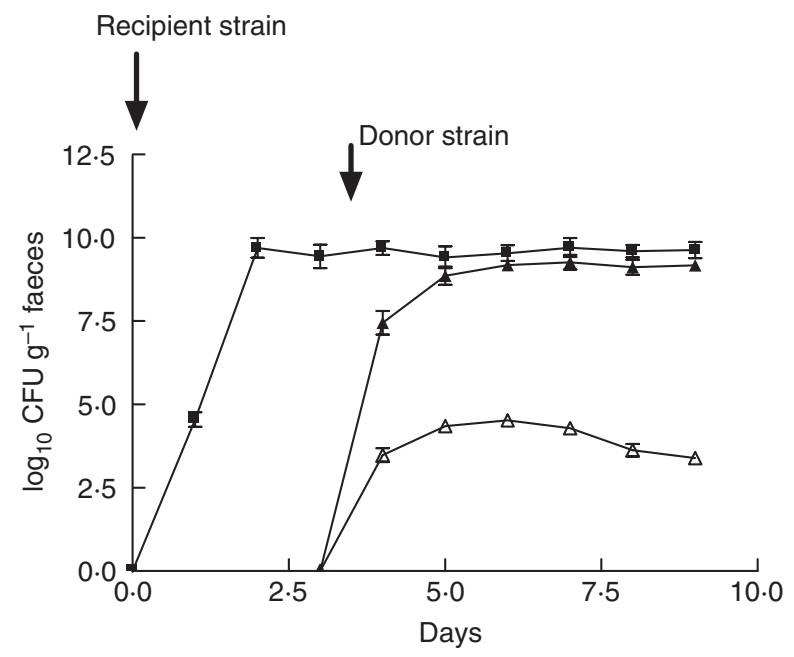

Figure 3 Bacterial counts in faeces of mice colonized with human faecal microbiota. Day 0, mice were inoculated with $10^{8}$ CFU Enterococcus faecalis $\mathrm{JH}_{2}$-SS (recipient strain), and on day 3 , mice were inoculated with $10^{8}$ CFU Ent. faecalis CS-19 (donor strain). (ロ) Entero-

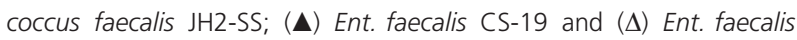
transconjugants. Data are the mean of $\log _{10}$ CFU g ${ }^{-1}$ faeces. The error bars represent the standard deviation.

ranging from $10^{3}$ to $10^{4} \mathrm{CFU} \mathrm{g}^{-1}$ faeces (Fig. 3). Similar numbers $\left(10^{3} \mathrm{CFU} \mathrm{g}^{-1}\right.$ tissue $)$ of transconjugant strains were also observed in mice intestines obtained by postmortem at the end of the experiment. Figure 4 illustrates the similar electrophoretic patterns obtained for the wildtype (lane 1) and transconjugated (lane 2) Ent. faecalis JH2-SS strains and the differing profile of the donor strain (Ent. faecalis CS19) (lane 3).

\section{Discussion}

The emergence of high-level gentamicin resistance (HLRG) in clinical medicine in 1979 had a substantial negative effect on the treatment for severe enterococcal infections and was rapidly followed by many reports of nosocomial infection in the 1980s (Zervos et al. 1987).

Antibiotic pressure may not only affect the microbiota, but also promote the transfer of elements encoding resistance to the particular drug. The transfer (by mobile genetic elements) of genes conferring ATM resistance
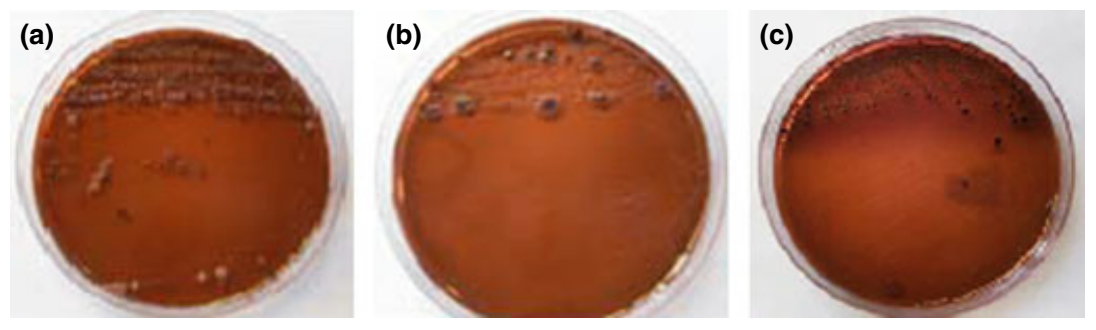

Figure 2 Mice faeces microbiota isolation in EMB-Levine agar pre-ceftriaxone (a), $24 \mathrm{~h}$ after two doses of ceftriaxone, the microbiota changed (b), and $24 \mathrm{~h}$ postadministration of $\mathrm{HFc}$, mice intestine with human-microbiota colonization (c). HFc, human faece. 


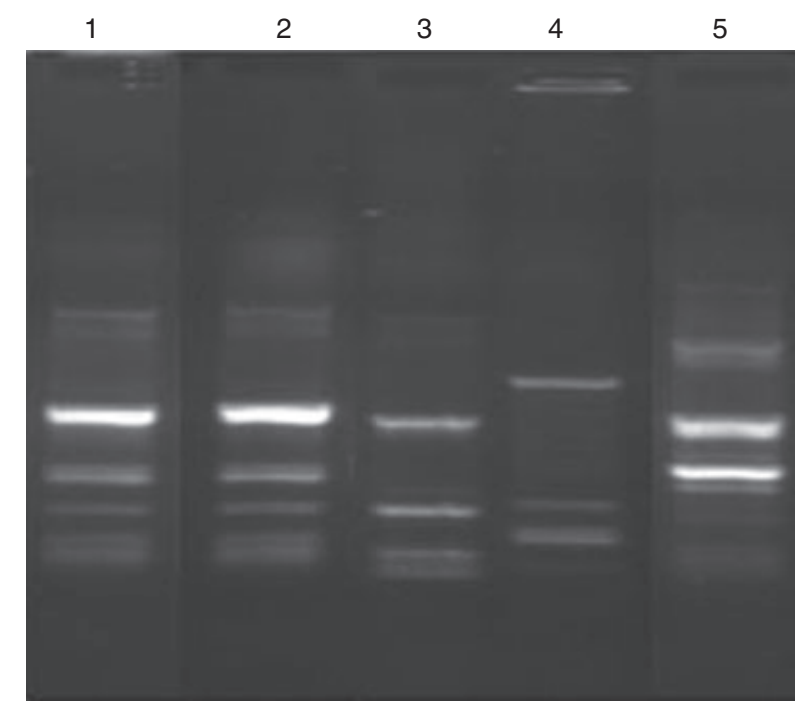

Figure 4 RAPD-PCR Enterococcus faecalis strain from faeces. Lane 1: Ent. faecalis JH2-SS; lane 2: Ent. faecalis JH2-SS transconjugated; lane 3: Ent. faecalis CS19; lane 4: Enterococcus faecium ATCC 19434 (control 1); lane 5: Ent. faecalis CS26 (control 2).

from the strains obtained from food samples has been reported (Hayes et al. 2004). However, plasmid conjugation provides a more efficient way for horizontal gene transfer and is generally considered the most relevant mechanism for the increase in bacteria multiresistant to conventional ATMs (Kurenbach et al. 2003).

Higher levels of intestinal colonization by Ent. faecalis are a frequent precursor of human invasive infections, although other predeterminants may be important in patients with comorbidity factors. One reason for this may be the failure of local or systemic use of ATM to remove Ent. faecalis owing to its natural antibiotic resistance (i.e. to cephalosporins). The use of broad-spectrum ATM contributes to the development of a selective genetic pressure on the intestinal microbiota, resulting in the increased emergence of multiresistant bacteria. The human GI tract is a massive reservoir of bacteria with the potential of acquiring and transferring resistance to conventional ATMs. In addition, there is the possibility that bacteria isolated from the food of animal origin may act as a pool of resistance. Recently, it has been reported that $15 \%$ of enterococci isolated from the faeces of hospitalized patients exhibit HLRG (Ronconi and Merino 2000).

The in vitro findings detailed in this paper are consistent with a previous study (Clewell 1990) in which the author noted the ability of enterococci to exchange genetic information by conjugation and with another (Huycke et al. 1992) study which, based on studies in a hamster model, reported that such exchange processes may occur in the gastrointestinal tract.
The design of in vivo models to assess the gene transfer has proved challenging but such an approach is needed in order to provide definitive evidence of genetic exchange between enterococcal strains from the food of animal origin and humans. An in vivo model that reproduced the interaction of the food strains with the human GI tract microbiota, which involved colonizing gnotobiotic mice with human colonic microbiota, was reported by Hirayama (1999). More recently, a model for assessing the transfer of vancomycin resistance from gen vanA obtained from an animal isolate to one from clinical human source in the intestine of mice treated with cefuroxime has been reported by Lester and Hammerum (2010). There have been consistent reports on the in vivo transfer of glycopeptide resistance along with the transfer of gen vanA and other resistance genes from porcine to human Enterococcus faecium at high frequency in the digestive tract of gnotobiotic mice (Moubareck et al. 2003; Dahl et al. 2007,). However, there have been no previous in vivo reports for the transfer of HLRG in an environment comparable to the human intestine. In the present study, a model for genetic transfer in nonsterile mice colonized with human GI microbiota was established. The results obtained suggest that the gastrointestinal environment is important for gene flux both within and between transient and resident bacterial species. Moreover, the colonization of the GI tract by indigenous Ent. faecalis strains containing HLRG implies the possibility of translocation.

Donabedian et al. (2003) evaluated gentamicin-resistant enterococci isolated from humans, food and farm animals and provided evidence for the spread of gentamicin-resistant enterococci from animals to humans through the food supply. They showed that enterococci isolated from animals and humans possess the same aminoglycoside resistance genes. However, it is significant to determine the transferability of these resistance genes because the dissemination of genes can occur by horizontal transfer.

The current experiments provide the first evidence of the possibility of HLRG gene transfer between enterococci from the food of animal origin to human enterococci in a similar environment with immune-competent mice colonized with human microbiota.

Furthermore, phenotyping of the most common enterococci in the intestine of human volunteers has revealed that the enterococcal microbiota of the human intestine often changes suggesting that, in general, enterococci do not colonize for extended periods of time (Gelsomino et al. 2003). However, the transitory colonization of resistant enterococci strains such as those with HLRG, as confirmed by the in vivo results described in this paper, may be sufficient to allow gene transfer. In the current studies, it is relevant to note that colonization of the intestine with human microbiota persisted for at least 
10 days and therefore offers a useful in vivo technique for studying the genetic transfer in a human bowel model (Fig. 3).

In conclusion, the in vivo genetic transfer of HLRG from strains isolated from the food of animal origin to human faecal microbiota has been confirmed within a mouse model. The complexity found in the therapeutic responses of invasive infectious diseases associated with Ent. faecalis allows the assessment of food of animal origin as a reservoir for resistant pathogenic bacteria. In addition, this study may contribute to the understanding of ATM resistance gene transfer between Ent. faecalis strains from food and the human GI tract.

\section{References}

Castro, M., Sparo, M., Molina, M., Andino, J. and Manghi, M. (2007) Enterococcus faecalis CECT7121 induces systemic immunomodulatory effects and protects from Salmonella infection. Int J Probiotics Prebiotics 2, 215-224.

Cave, H., Bingen, E., Elion, J. and Denamur, E. (1994) Differentiation of Escherichia coli strains using randomly amplified polymorphic DNA analysis. Res Microbiol 145, 141-150.

Clewell, D.B. (1990) Movable genetic elements and antibiotic resistance in enterococci. Eur J Microbiol Infect Dis 9, 90102.

Dahl, K.H., Mater, D., Flores, M., Johnsen, P., Midtvedt, T., Corthier, G. and Sundsfjord, A. (2007) Transfer of plasmid and chromosomal glycopeptide resistance determinants occurs more readily in the digestive tract of mice than in vitro and exconjugants can persist stably in vivo in the absence of glycopeptide selection. J Antimicrob Chemother 59, 478-486.

Donabedian, S.M., Thal, L.A., Hershberger, E., Perri, M.B., Chow, J.W., Bartlett, P., Jones, R., Joyce, K. et al. (2003) Molecular characterization of gentamicin-resistant Enterococci in the United States: evidence of spread from animals to humans through food. J Clin Microbiol 41, 11091113.

Ferretti, J.J., Gilmore, K. and Courvalin, P. (1986) Nucleotide sequence analysis of the gene specifying the bifunctional 6'-aminoglycoside acetyltransferase 2"'-aminoglycoside phosphotransferase enzyme in Streptococcus faecalis and identification and cloning of gene regions specifying the two activities. J Bacteriol 167, 631-638.

Foulquié, M.R., Sarantinopoulos, P., Tsakalido, E. and De Vuyst, L. (2006) The role and application of enterococci in food and health. Int J Food Microbiol 106, 1-24.

Gelsomino, R., Vancanneyt, M., Cogan, T.M. and Swings, J. (2003) Effect of raw-milk cheese consumption on the enterococcal flora of human feces. Appl Environ Microbiol 69, 312-319.

Gentile, J.H., Sparo, M.D., Pipo, V.B. and Gallo, A.J. (1995) Meningitis a Enterococcus faecalis. Medicina 55, 435-437.
Hayes, J., English, L., Carr, L., Wagner, D. and Joseph, S. (2004) Multiple-antibiotic resistance of Enterococcus spp. isolated from commercial poultry production environments. Appl Environ Microbiol 70, 6005-6011.

Hirayama, K. (1999) Ex-germ free mice harboring intestinal microbiota derived from other animal species as an experimental model for ecology and metabolism of intestinal bacteria. Exp Anim 48, 219-227.

Huycke, M., Gilmore, M., Jett, B. and Booth, J. (1992) Transfer of pheromone-inducible plasmids between Enterococcus faecalis in the Syrian hamster gastro-intestinal tract. J Infect Dis 166, 1188-1191.

Kurenbach, B., Bohn, C., Prabhu, J., Abudukerim, M., Szewzyk, U. and Grohmann, E. (2003) Intergeneric transfer of the Enterococcus faecalis plasmid pIP501 to Escherichia coli and Streptomyces lividans and sequence analysis of its tra region. Plasmid 50, 86-93.

Leclercq, R. (1994) Enterococci acquire new kinds of resistance. Clin Infect Dis 24(Suppl 1), 80-84.

Lester, C. and Hammerum, A. (2010) Transfer of vanA from an Enterococcus faecium isolate of chicken origin to a CC17 E. faecium isolate in the intestine of cephalosporin-treated mice. J Antimicrob Chemother 65, 1534-1548.

Maschieto, A., Martinez, R., Vanzato, I. and Da Costa, A. (2004) Antimicrobial resistance of Enterococcus sp. isolated from the intestinal tract of patients from a University Hospital in Brazil. Mem Inst Oswaldo Cruz 99, 763767.

Moellering, R.C. Jr, Wennersten, C., Medrek, T. and Weinberg, A.N. (1970) Prevalence of high-level resistance to aminoglycosides in clinical isolates of enterococci. Antimicrob Agents Chemother 10, 335-340.

Moubareck, C., Bourgeois, N., Courvalin, P. and Doucet-Populaire, F. (2003) Multiple antibiotic resistance gene transfer from animal to human enterococci in the digestive tract of gnotobiotic mice. Antimicrob Agents Chemother 47, 2993 2996.

Predari, S.C., Gutierrez, M.A., Ribas, C., Molinari, G.S. and Santoiani, J.E. (1999) Susceptibility of Enterococcus faecalis to twelve antibiotics, time kill assays, and high level aminoglycoside resistance in University Hospital in Argentina. Rev Argent Microbiol 2, 67-70.

Ronconi, M.C. and Merino, L.A. (2000) Prevalencia de Enterococcus faecalis y E. faecium con resistencia de alto nivel a aminoglucósidos en las ciudades de Resistencia y Corrientes, Republic of Argentina. Enferm Infecc Microbiol Clin 18, 71-73.

Ruiz-Barba, J.L., Piard, J.C. and Jimènez-Díaz, R. (1991) Plasmid profiles and curing of plasmids in Lactobacillus plantarum strains isolated from green olive fermentations. $J$ Appl Bacteriol 71, 417-421.

Sparo, M. and Mallo, R. (2001) Evaluación de la flora bacteriana de un ensilado natural de maíz. Rev Argent Microbiol $33,75-80$. 
Sparo, M.D., Castro, M.S., Andino, P.J., Lavigne, M.V., Ceriani, C., Gutiérrez, G.L., Fernández, M.M., De Marzi, M.C. et al. (2006) Partial characterization of enterocin MR99 from a corn silage isolate of Enterococcus faecalis. J Appl Microbiol 100, 123-134.

Sparo, M., Nuñez, G.G., Castro, M., Calcagno, M.L., Garcia Allende, M.A., Ceci, M., Najle, R. and Manghi, M. (2008) Characteristics of an environmental strain, Enterococcus faecalis CECT7121, and its effects as additive on craft dryfermented sausages. Food Microbiol 25, 607-615.

Yagi, Y. and Clewell, D.B. (1980) Recombination-deficient mutant of Streptococcus faecalis. J Bacteriol 143, 966-970.

Zervos, M., Kauffman, C., Therasse, P., Bergman, A., Mikesell, T. and Schaberg, D. (1987) Nosocomial Infection by gentamicin-resistant Streptococcus faecalis: an epidemiologic study. Ann Intern Med 106, 687-691. 